\title{
Materiais para Eletrodos que Emitem Termionicamente em Soldagem a Arco
}

\section{(Materials for Thermionically Emitting Electrodes in Arc Welding)}

\author{
Juliane Ribeiro da Cruz ${ }^{1}$, Américo Scotti ${ }^{1}$ \\ 1Universidade Federal do Paraná, Departamento de Engenharia Mecânica, Curitiba,PR,Brasil,juliane.rcruz@gmail.com, scotti@ufpr.br
}

\section{Resumo}

Eletrodos não consumiveis são feitos de tungstênio puro ou ligado, para processos a arco, e de grafita, para goivagem. Contudo, na literatura técnica dedicada à soldagem a arco encontram-se poucas considerações acerca da não utilização de outros materiais como emissores termiônicos. Com o desafio de contribuir para o preenchimento dessa lacuna, procurou-se justificar a inviabilidade da utilização do ferro puro na fabricação de eletrodos não consumiveis e demonstrar o potencial de emissão termiônica de eletrodos de tungstênio puro ou com tória e da grafita. Inicialmente, foram determinadas teoricamente as áreas de emissão, e os respectivos diâmetros, necessárias para soldar com 100 e $300 \mathrm{~A}$ em várias temperaturas. Posteriormente, foram calculadas as temperaturas máximas alcançadas por efeito Joule nesses eletrodos em seus diferentes diâmetros. A validação dos resultados se deu por confrontamento com os fenômenos observados na prática. Eletrodos não consumiveis de ferro puro mostram-se inviáveis, pois precisariam ter diâmetro excessivamente grande para emitir em níveis usuais de corrente abaixo da sua temperatura de fusão. Além disso, nesses diâmetros eles não aqueceriam por efeito Joule até a temperatura de emissão. Por outro lado, demonstrou-se que eletrodos de tungstênio puro e dopado e de grafita emitem com diâmetros reduzidos em temperaturas próximas a de fusão/sublimação e alcançam as temperaturas de emissão rapidamente por efeito Joule. Apesar das simplificações adotadas, também ficou justificada a necessidade de afiação dos eletrodos para soldagem a arco e demonstrado que a dopagem de eletrodos de $W$ com óxidos de menor função-trabalho limita o aquecimento da ponta do eletrodo evitando sua fusão superficial.

Palavras-chave: Emissão termoiônica. Equação de Richardson-Dushman. Efeito Joule. Eletrodo não consumível. Afiação do eletrodo.

\begin{abstract}
Nonconsumable electrodes are made of tungsten and its alloys, for arc welding processes, and of graphite, for arc gouging. However, current technical literature does not give detailed explanations for not using other materials as thermionic emitters. Taking the challenge of contributing to the fulfillment of this knowledge gap, this study attempts to justify the unviable use of pure iron as a nonconsumable electrode material and to demonstrate the thermionic emission capacity of pure and thoriated tungsten and graphite electrodes. Initially, the emission areas, and the respective electrodes diameters, for welding at 100 and $300 \mathrm{~A}$ at several temperatures were theoretically determined. Then, the maximum temperatures reached by different diameters electrodes due to Joule effect were calculated. The outcomes validation was carried out by confronting them to the phenomena observed in practice. Nonconsumable iron electrodes showed to be unviable, because they would require extremely large diameters to emit in usual current levels below their melting temperatures. Besides, they would not be heated by Joule effect up to the emission temperature before arc extinction. On the other hand, it has been demonstrated that pure and alloyed tungsten and graphite electrodes emit with small diameters approaching their melting/ sublimation temperatures and they promptly reach the emission temperatures by Joule effect. Despite the adopted simplifications, the electrodes sharpening need for arc welding was also justified, as well as it was demonstrated that the $W$ electrode doping with lower work-function oxides limit the heating of the electrode preventing its superficial melting.
\end{abstract}

Key-words: Thermionic emission. Richardson-Dushman equation. Joule effect. Nonconsumable electrode. Electrode grinding.

\section{Introdução}

Em processos de soldagem a arco com eletrodos não consumíveis, por exemplo, o TIG, se utilizam eletrodos feitos

Recebido em 12/08/2013, texto final em 13/09/2013. de materiais refratários que suportam a temperatura de trabalho e mantém sua integridade. Quando esses eletrodos estão na polaridade negativa (terminologia antigamente referenciada como polaridade direta), o eletrodo emite elétrons a temperaturas suficientemente altas por um mecanismo chamado de emissão termiônica. A emissão de elétrons por esse mecanismo demanda menos energia para acontecer, se comparado à emissão por campo (que ocorre em materiais não refratários), resultando em menor deterioração de eletrodo. $\mathrm{O}$ aquecimento dos eletrodos não consumíveis inicia-se com a abertura do arco, seja por 
alta tensão e alta frequência, em que um pulso de tensão ioniza o meio entre o eletrodo e a peça, ou por curto-circuito com corrente controlada, em que o baixo valor de corrente é suficiente para aquecer a ponta do eletrodo até a condição da emissão termiônica. Juntamente com o arco, surgem possíveis fontes de calor para maior aquecimento do eletrodo e facilitação da emissão termiônica. Dentre essas fontes estariam, segundo Cheng [1], o efeito Joule, a radiação do arco, a energia das reações exotérmicas na superfície do eletrodo e as energias térmica e cinética dos íons que se chocam contra o cátodo.

Lima [2] e Andrade [3] citam que o efeito termiônico é observado quando elétrons escapam do material ao serem excitados com uma energia térmica superior à sua funçãotrabalho, que é a energia mínima para remoção de um elétron do material. Esse efeito leva ao aparecimento de uma nuvem eletrônica ao redor do material, que dificulta ou impede a saída de outros elétrons. Para esses autores, caso uma diferença de potencial seja aplicada entre o cátodo e o ânodo de um material nessa condição, inicia-se a passagem da corrente termiônica e elimina-se a nuvem e sua influência na emissão de novos elétrons.

Entretanto para haver emissão termiônica, não é necessário estar em alta temperatura. Conforme observado por Richardson [4], um corpo emite termionicamente um número de elétrons limitado por área em função de sua temperatura e material. Quanto menor a temperatura, maior a área necessária para se emitir uma mesma quantidade de elétrons por efeito termiônico. Eletrodos não consumíveis para processos a arco são feitos de tungstênio puro ou de ligas de tungstênio com certos óxidos (tória, zircônia, de lantânio, dentre outros) que facilitam a emissão termiônica dos eletrodos quando colocados no polo negativo. Acredita-se que a grafita, usada como eletrodo na goivagem a arco, também emita pelo mecanismo termiônico. Caso outros materiais para eletrodos, mesmo consumíveis, emitissem elétrons (corrente) em intensidade suficiente (ou um percentual significativo) por efeito termiônico, poderiam ser esperados comportamentos diferenciados nos processos de soldagem já existentes, como uma menor tensão de soldagem no processo MIG, por exemplo, ou maior densidade de corrente no arco em soldagem TIG de alumínio durante a polaridade positiva.

Por outro lado, a prática ao longo dos anos mostra que em eletrodos consumíveis o mecanismo de emissão predominante não é o termiônico (a emissão é dita ser por campo). Guile [5] e Lancaster [6] justificam que materiais com baixo ponto de ebulição são incapazes de atingir temperaturas suficientemente altas para que a emissão termiônica tenha um papel significativo na intensidade de corrente produzida. Além das justificativas, não se encontrou na literatura técnica dedicada à soldagem a arco estudos que demonstrassem a inviabilidade da utilização de materiais não refratários como emissor termiônico, mesmo em temperaturas menores a custa de maiores áreas de emissão. Tampouco, estudos que justificassem a necessidade da afiação do eletrodo para abertura e manutenção do arco no processo TIG. De fato, o que mais se encontra é a afirmação de que a emissão termiônica ocorre somente em materiais refratários, o que contraria em parte relatos, por exemplo, no trabalho de
Richardson [4], de que diversos materiais ( $\mathrm{Fe}, \mathrm{C}, \mathrm{Pt}, \mathrm{Na}$, metais alcalino-terrosos) poderiam emitir termionicamente.

Porém, demonstrar a fenomenologia da emissão termiônica em soldagem a arco é uma tarefa muito complexa, tanto teoricamente como experimentalmente. Dessa forma, torna-se um desafio tecnológico encontrar, de uma forma mais simples e acessível, um melhor entendimento do fenômeno que governa a emissão termiônica por meio dos materiais utilizados na confecção de eletrodo de soldagem a arco. Assim, esse trabalho tem como objetivos propor uma justificativa para a inviabilidade da utilização de eletrodos não consumíveis a base de ferro puro, demonstrar teoricamente a capacidade de emissão termiônica de eletrodos à base de tungstênio e grafita, verificar o efeito do ângulo de afiação do eletrodo sobre a facilidade de emissão e comparar as características de emissão de eletrodos de tungstênio puro e de tungstênio dopado com $2 \%$ de tória.

\section{Metodologia}

Para contemplar o objetivo desse trabalho, tomou-se como primeira base metodológica a análise dos diâmetros de eletrodo necessários para emissão termiônica correspondente a uma determinada corrente para três materiais (W, Fe e grafita). Usou para tal a conhecida equação de Richardson-Dushman (Equação 1), que quantifica a densidade de corrente produzida por efeito termiônico. Com base nessa equação, pode-se determinar o diâmetro necessário para que um material emita com uma dada intensidade de corrente numa certa temperatura. Nessa equação, J é a densidade de corrente (A. $\left.\mathrm{m}^{-2}\right)$, A é uma constante termiônica $\left(\mathrm{A} \cdot \mathrm{K}^{-2} \cdot \mathrm{m}^{-2}\right)$, que depende do material, da temperatura e da contaminação da superfície do eletrodo, $\mathrm{T}$ é a temperatura do eletrodo (K), e é a carga do elétron (C), $\Phi$ é a função-trabalho do material em função da temperatura $(\mathrm{eV})$ e $\mathrm{k}_{\mathrm{B}}$ é a constante de Boltzmann (J.K-1). Por essa equação, quanto maior a temperatura, maior a densidade de corrente possível de se alcançar por efeito termiônico. Entretanto, a temperaturas próximas da ambiente, um material também emitiria termionicamente, mas com densidade de corrente muito baixa. Porém, não se utilizam baixas densidades de corrente em processos de soldagem a arco, devido a seus fatores intrínsecos.

$J=A T^{2} \exp ^{\left(\frac{-e \Phi}{k_{B} T}\right)}$

Além disto, no caso de soldagem a arco, o eletrodo não é aquecido por fontes externas de calor, sendo aquecido pelo calor gerado no próprio arco (acoplamento arco-eletrodo) e por efeito Joule (ambos aumentando de intensidade com a corrente de soldagem).

Então, a segunda base metodológica deste trabalho é que o eletrodo (material e diâmetro) teria que se aquecer como resultado da corrente de soldagem para poder emitir termionicamente na temperatura de trabalho. Para simplificar esta análise complexa, assumiu-se que o aquecimento em questão (até a temperatura para emissão) se daria somente por efeito Joule. Esta hipótese pode ser considerada como verdadeira particularmente quando se considera a emissão logo após a abertura do arco, desprezando o efeito do aquecimento provocado pelo arco após 
seu acendimento pleno. Acredita-se que estas simplificações deixem a análise apenas mais conservadora, uma vez que, se um eletrodo de um dado diâmetro emite termionicamente logo após abertura do o arco, ele emitirá ainda com maior facilidade com o aumento do calor recebido pelo arco após acendimento pleno.

\subsection{Determinação do diâmetro do eletrodo para emissão ter- miônica}

Inicialmente foram determinados, em função da densidade de corrente de trabalho, os diâmetros que eletrodos afiados de tungstênio, grafita e ferro puro precisariam para emitir elétrons na intensidade de corrente desejada em dadas temperaturas, usando-se a equação de Richardson-Dushman (Equação 1). Por definição, a densidade de corrente, $\mathrm{J}\left(\mathrm{A} \cdot \mathrm{m}^{-2}\right)$, é a razão entre a corrente de soldagem, I (A), e a área de emissão do eletrodo, $\mathrm{S}\left(\mathrm{m}^{2}\right)$, Equação 2. Assumiu-se a área de emissão como sendo a área lateral de um cone de base circular, Figura 1, expressa pela Equação 3, em função do ângulo de afiação do eletrodo, $\alpha\left(^{\circ}\right)$, e do diâmetro do eletrodo, $\mathrm{d}(\mathrm{mm})$. Assumir que a emissão ocorra por toda a área lateral cone é razoável, pois se procura o menor diâmetro do eletrodo para produção da corrente desejada (diâmetro da base do cone). Na prática são usados eletrodos de diâmetros maiores do que o mínimo e, neste caso, a emissão deixa de ocorrer em todo o cone e se concentra apenas na extremidade.

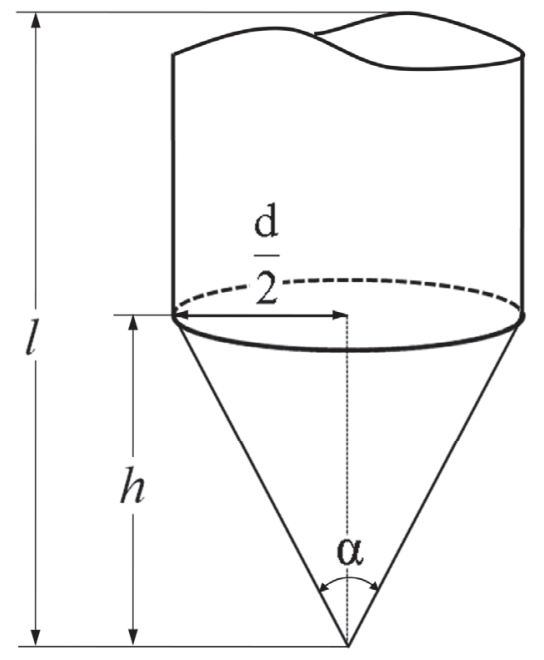

Figura 1. Representação de um eletrodo afiado, cuja área assumida para emissão é a área lateral de um cone de base circular

Substituindo (3) em (2) e isolando-se o diâmetro, d (m), temse a equação para o cálculo do diâmetro $(\mathrm{mm})$ em função da corrente e densidade de corrente, Equação 4.

$$
J=\frac{I}{S}
$$

$S=\frac{\pi d^{2}}{4 \operatorname{sen}(\alpha / 2)}$ $d=1000 \sqrt{\frac{4 I \operatorname{Isen}(\alpha / 2)}{J \pi}}$

O cálculo do diâmetro de cada eletrodo para emissão na intensidade de corrente desejada foi feito usando-se duas temperaturas limites, a de fusão e a de ebulição. Apesar de ser assumido que toda área da superfície de emissão esteja exposta a uma mesma temperatura (fusão ou ebulição), não quer dizer que todo o volume da ponta do eletrodo esteja fundido ou em ebulição, podendo o eletrodo ainda assim ser considerado não consumível. A simulação foi feita para dois valores de corrente, a saber, 100 e $300 \mathrm{~A}$. Esses valores foram escolhidos por delimitarem uma gama usual de corrente de trabalho no processo TIG. Além da temperatura limite e da corrente, o ângulo de afiação do eletrodo também foi usado como parâmetro de entrada nos cálculos, por alterar a área de emissão e, por consequência, o diâmetro mínimo do eletrodo para emissão. $\mathrm{O}$ ângulo de afiação, para fins de cálculos, assumiu valores de 30, 60,90 e $180^{\circ}$. As temperaturas de fusão/sublimação e ebulição, propriedades dos materiais e constantes utilizadas nos cálculos estão listada na Tabela 1.

Pela literatura corrente, verificou-se que para um mesmo material são atribuídos valores muito diferentes para a constante termiônica. Por exemplo, a constante termiônica do tungstênio assume os valores empíricos de $6,00 \mathrm{E}+05[1,7]$ e de $7,00 \mathrm{E}+05$ [13], o valor teórico de 1,20E+06 [2,3] e valores de até 6,00E+09 em vácuo [16]. No presente trabalho, atribui-se à constante termiônica o valor experimental comum de $6,00 \mathrm{E}+05 \mathrm{~A} \cdot \mathrm{K}^{-2} \cdot \mathrm{m}^{-2}$, com a função-trabalho do material variando com a temperatura, Equação 5, conforme a abordagem recomendada por Dushman [5]. Ainda na Equação 5, $\Phi(\mathrm{eV})$ é a função-trabalho em função da temperatura, $\Phi_{0}(\mathrm{eV})$ é a função-trabalho na temperatura ambiente (Tabela 1) e $\mathrm{T}(\mathrm{K})$ a temperatura de emissão, a qual neste trabalho assumiu-se variar de 1500 a aproximadamente $6000 \mathrm{~K}$. É importante ressaltar que a utilização do menor valor da constante termiônica tende a resultar em menor densidade de corrente (até mesmo com a correção da função-trabalho, que diminui com o aumento da temperatura) e, consequentemente, maior diâmetro do eletrodo para produção da intensidade de corrente desejada. Assim, o presente cálculo considerou a condição mais desfavorável de emissão, de forma que a utilização de constantes com valores maiores resultariam em menor diâmetro de eletrodo necessário para emissão.

$\Phi=\Phi_{0}-\frac{3}{2} \frac{k_{B} T}{e}$

\subsection{Aquecimento máximo do eletrodo por efeito Joule}

O calor gerado pelo efeito Joule $\left(\mathrm{Q}_{\mathrm{EJ}}\right)$ em um eletrodo pode ser expresso pela Equação 6, em que $\mathrm{R}$ é a resistência elétrica $(\Omega)$, I é a corrente elétrica (A) e t é o tempo de passagem de corrente (s). A resistência elétrica, por sua vez, é função da resistividade, $\rho(\Omega . \mathrm{m})$, do comprimento do eletrodo, $1(\mathrm{~m})$, e da área da seção transversal, $\mathrm{S}_{\mathrm{T}}\left(\mathrm{m}^{2}\right)$, Equação 7. O aquecimento dos eletrodos foi simulado para um valor arbitrário de $1 \mathrm{~s}$ para representar o tempo de inicio do arco. 
Tabela 1. Temperaturas de fusão/sublimação e ebulição, propriedades dos materiais e constantes utilizadas na aplicação da equação de Richardson-Dushman para o cálculo do diâmetro do eletrodo

\begin{tabular}{|c|c|c|c|c|c|c|}
\hline & $\mathrm{A}\left(\mathrm{A} \cdot \mathrm{K}^{-2} \cdot \mathrm{m}^{-2}\right)$ & Fusão* (K) & Ebulição (K) & $\Phi_{0}(\mathrm{eV})$ & e (C) & $\mathbf{k}_{\mathrm{B}}\left(\mathbf{J} . \mathbf{K}^{-1}\right)$ \\
\hline $\mathbf{W}^{[7,8]}$ & $6,00 \mathrm{E}+05$ & 3640 & 6170 & 4,530 & \multirow{4}{*}{$1,6 \mathrm{E}-19$} & \multirow{4}{*}{$1,38 \mathrm{E}-23$} \\
\hline Grafita $^{[9,10]}$ & $6,00 \mathrm{E}+05$ & 3920 & - & 4,620 & & \\
\hline $\mathbf{F} \mathbf{e}^{[11,12]}$ & $6,00 \mathrm{E}+05$ & 1810 & 3134 & 4,310 & & \\
\hline $\mathrm{W}+2 \% \mathrm{ThO}_{2}^{[13,14]}$ & $6,00 \mathrm{E}+05$ & 3660 & 4670 & 2,700 & & \\
\hline
\end{tabular}

*Temperatura de sublimação para a grafita

$Q_{E J}=R I^{2} t$

$R=\frac{\rho l}{S_{T}}$

A temperatura máxima alcançada pelo eletrodo por efeito Joule foi determinada com o auxílio da equação do calor sensível, Equação 8, em que Q é o calor sensível (J), m é a massa do eletrodo $(\mathrm{kg})$, c é o calor específico do material do eletrodo $\left(\mathrm{J} . \mathrm{kg}^{-1} \cdot \mathrm{K}^{-1}\right)$ e $\Delta \mathrm{T}$ é a diferença entre as temperaturas inicial, $\mathrm{T}_{\mathrm{i}}$ $(\mathrm{K})$, e final, $\mathrm{T}_{\mathrm{f}}(\mathrm{K})$.

$Q=m c \Delta T$

Assumindo-se o aquecimento do eletrodo somente por efeito Joule, pode-se igualar as Equações 6 e 8, tendo como resultado a Equação 9.

$R I^{2} t=m c \Delta T$

A massa do eletrodo, m $(\mathrm{kg})$, Equação 10, é representada pelo produto entre sua densidade, $\sigma\left(\mathrm{kg} \cdot \mathrm{m}^{-3}\right)$, e seu volume, $\mathrm{V}$ $\left(\mathrm{m}^{3}\right)$, o qual é a soma do volume da região cilíndrica com o da região cônica do eletrodo. $\mathrm{O}$ volume da parte cilíndrica pode ser expresso pela multiplicação da área da sua seção transversal, $\mathrm{S}_{\mathrm{T}}$ $\left(\mathrm{m}^{2}\right)$, pelo seu comprimento, o qual é dado pela subtração do comprimento total do eletrodo, 1 (m), da altura do cone, $\mathrm{h}(\mathrm{m})$, Figura 1. O volume da parte cônica, por sua vez, é calculado pelo produto da área da sua seção transversal por um terço da altura do cone.

$$
m=\sigma V=\sigma\left[S_{T}(l-h)+\frac{1}{3} S_{T} h\right]
$$

Expressando a altura do cone em função do diâmetro do eletrodo, a massa do eletrodo pode ser expressa pela Equação 11.

$m=\sigma S_{T}\left[l-\frac{d}{3 \operatorname{tg}(\alpha / 2)}\right]$

Substituindo as equações (7) e (11) em (9) e rearranjando para a temperatura final $\mathrm{T}_{\mathrm{f}}(\mathrm{K})$ chega-se à Equação 12 .

$$
T_{f}=\frac{\rho l I^{2} t}{\sigma c S_{T}^{2}\left[l-\frac{d}{3 \operatorname{tg}(a / 2)}\right]}+T_{i}
$$

Substituindo-se a área da seção transversal, $\mathrm{S}_{\mathrm{T}}\left(\mathrm{m}^{2}\right)$, da Equação 13, em (12), tem-se a temperatura final do eletrodo em função do comprimento do eletrodo, corrente, tempo de aquecimento, ângulo de afiação, diâmetro do eletrodo, propriedades do material e temperatura inicial, Equação 14.

$$
\begin{aligned}
& S_{T}=\frac{\pi d^{2}}{4} \\
& T_{f}=\frac{4^{2} \rho l I^{2} t}{\sigma c \pi^{2} d^{4}\left[l-\frac{d}{3 \operatorname{tg}(a / 2)}\right]}+T_{i}
\end{aligned}
$$

Foram calculadas as temperaturas máximas alcançadas por eletrodos de diâmetros variáveis a fim de verificar se apenas o aquecimento resistivo seria suficiente para elevar a temperatura desses eletrodos até sua respectiva temperatura de emissão. No cálculo do aquecimento por efeito Joule, foram considerados eletrodos com ângulo de ponta de $30^{\circ}$, pois possuem as maiores áreas de emissão dentre os ângulos de ponta estudados, portanto resultando em menores diâmetros de emissão. Foram utilizadas as propriedades dos materiais da Tabela 2, corrente de $100 \mathrm{~A}$, tempo de aquecimento de $1 \mathrm{~s}$, temperatura inicial de $300 \mathrm{~K} \mathrm{e}$ comprimento do eletrodo de $25 \mathrm{~mm}$, por ser um comprimento de eletrodo usual em soldagem TIG.

Deve-se destacar que, no cálculo do aquecimento por efeito Joule, desconsiderou-se o aumento do aquecimento resistivo com a redução da área da seção transversal na ponta do eletrodo (região cônica). Assumiu-se que a temperatura de emissão é homogênea em toda a área superficial do cone e é equivalente à temperatura alcançada por efeito Joule na base do cone (cuja área da seção transversal é a mesma do eletrodo). Na prática sabe-se que há um gradiente de temperatura na área de emissão e que cada região contribui com densidades de correntes diferentes na geração da corrente total. Esta simplificação (de que a temperatura de emissão é a menor temperatura encontrada na ponta do eletrodo) assegura que está se considerando a condição mais desfavorável de emissão, pois, caso o gradiente de temperatura na ponta do eletrodo fosse considerado, as densidades de corrente seriam maiores e diâmetro de eletrodo calculado para produção da corrente desejada seria menor. 
Tabela 2. Propriedades dos materiais estudados e condições de simulação

\begin{tabular}{|c|c|c|c|c|c|c|}
\hline & $\rho(\Omega . m)$ & $\sigma\left(\mathrm{kg} \cdot \mathrm{m}^{-3}\right)$ & c $\left(\mathbf{J} . \mathrm{kg}^{-1} \cdot \mathrm{K}^{-1}\right)$ & $t(s)$ & $\mathbf{T i}(\mathrm{K})$ & $1(\mathrm{~m})$ \\
\hline $\mathbf{W}^{[6,13]}$ & $5,65 \mathrm{E}-08$ & 19300 & $300^{*}$ & \multirow{4}{*}{1} & \multirow{4}{*}{300} & \multirow{4}{*}{0,025} \\
\hline Grafita $^{[8]}$ & $6,00 \mathrm{E}-05$ & 2250 & 708 & & & \\
\hline $\mathbf{F e}^{[10]}$ & $8,90 \mathrm{E}-08$ & 7870 & 440 & & & \\
\hline $\mathbf{W}+\mathbf{2 \%} \mathrm{ThO}_{2}^{[13]}$ & $5,88 \mathrm{E}-08$ & 18872 & $310^{*}$ & & & \\
\hline
\end{tabular}

${ }^{*}$ Calor especifico em temperaturas próximas da temperatura de fusão

Tabela 3. Densidade de corrente e diâmetro de eletrodo necessário para emissão termiônica a 100 e 300 A na temperatura de fusão/ sublimação

\begin{tabular}{|c|c|c|c|c|c|c|c|c|c|}
\hline & & \multicolumn{4}{|c|}{$\mathbf{1 0 0} \mathbf{A}$} & \multicolumn{5}{|c|}{$\mathbf{3 0 0} \mathbf{A}$} \\
\hline & $\mathbf{J}$ & $\mathbf{d}_{\mathbf{3 0}^{\circ}}$ & $\mathbf{d}_{\mathbf{6 0}^{\circ}}$ & $\mathbf{d}_{\mathbf{9 0}^{\circ}}$ & $\mathbf{d}_{\mathbf{1 8 0 ^ { \circ }}}$ & $\mathbf{d}_{\mathbf{3 0}^{\circ}}$ & $\mathbf{d}_{\mathbf{6 0}^{\circ}}$ & $\mathbf{d}_{\mathbf{9 0}^{\circ}}$ & $\mathbf{d}_{\mathbf{1 8 0}^{\circ}}$ \\
\hline $\mathbf{W}$ & $1,93 \mathrm{E}+07$ & 1,31 & 1,82 & 2,16 & 2,57 & 2,26 & 3,15 & 3,74 & 4,45 \\
\hline Grafita & $4,81 \mathrm{E}+07$ & 0,83 & 1,15 & 1,37 & 1,63 & 1,43 & 1,99 & 2,37 & 2,82 \\
\hline Fe & $9,01 \mathrm{E}+00$ & 1912 & 2658 & 3161 & 3759 & 3312 & 4604 & 5475 & 6510 \\
\hline
\end{tabular}

$J$ - Densidade de corrente $\left(A . \mathrm{m}^{-2}\right) ; \mathrm{d}$ - diâmetro de eletrodo $(\mathrm{mm})$

Tabela 4. Densidade de corrente e diâmetro de eletrodo necessário para emissão termiônica a 100 e 300 A na temperatura de ebulição

\begin{tabular}{|c|c|c|c|c|c|c|c|c|c|}
\hline & & \multicolumn{4}{|c|}{$\mathbf{1 0 0} \mathbf{A}$} & \multicolumn{5}{|c|}{$\mathbf{A 0 0}$} \\
\hline & $\mathbf{J}$ & $\mathbf{d}_{\mathbf{3 0}^{\circ}}$ & $\mathbf{d}_{\mathbf{6 0}^{\circ}}$ & $\mathbf{d}_{\mathbf{9 0}^{\circ}}$ & $\mathbf{d}_{\mathbf{1 8 0}^{\circ}}$ & $\mathbf{d}_{30^{\circ}}$ & $\mathbf{d}_{\mathbf{6 0}^{\circ}}$ & $\mathbf{d}_{\mathbf{9 0}^{\circ}}$ & $\mathbf{d}_{\mathbf{1 8 0}^{\circ}}$ \\
\hline $\mathbf{W}$ & $2,06 \mathrm{E}+10$ & 0,04 & 0,06 & 0,07 & 0,08 & 0,07 & 0,10 & 0,11 & 0,14 \\
\hline $\mathbf{F e}$ & $3,14 \mathrm{E}+06$ & 3,24 & 4,50 & 5,35 & 6,37 & 5,61 & 7,80 & 9,27 & 11,03 \\
\hline
\end{tabular}

$J$ - Densidade de corrente $\left(A \cdot \mathrm{m}^{-2}\right) ; d$ - diâmetro de eletrodo $(\mathrm{mm})$

\section{Resultados e Discussão}

\subsection{Efeito das simplificações no aquecimento e diâmetro do eletrodo}

É importante ressaltar que a determinação da temperatura final do eletrodo aquecido por efeito Joule por alguns instantes (1 s) após a abertura do arco foi feita de forma simplificada. Foram desconsideradas as variações dimensionais do eletrodo e das propriedades do material em função da temperatura e as perdas de calor para o ambiente e transformações de fase durante o aquecimento do eletrodo.

Caso fossem considerados os aumentos da resistividade e do volume do eletrodo com a temperatura, o aquecimento do eletrodo seria maior devido ao aumento da resistência elétrica e à redução da densidade, que é inversamente proporcional à temperatura final do eletrodo (Equação 12). Além disso, o aquecimento também seria facilitado caso fossem consideradas outras fontes de calor, como a radiação, o choque dos íons e as reações exotérmicas na superfície do eletrodo. Por outro lado, caso fossem considerados o aumento do calor específico com a temperatura (Equação 12), o calor consumido na transformação de fase e as perdas de calor para o ambiente durante o aquecimento, a temperatura final dos eletrodos seria inferior à encontrada nesses cálculos.

Além disto, assumiu-se a área de emissão como apenas a área lateral de um cone circular reto. Caso a emissão também ocorresse pela região cilíndrica do eletrodo, a área de emissão seria maior e o diâmetro do eletrodo poderia ser menor.

Desta forma, é difícil prever o efeito das simplificações usadas, pois algumas favorecem o aquecimento e outras não. Assim, como se verá mais adiante, a validade dos resultados apresentados neste trabalho se fará baseado na ordem de grandeza e não necessariamente nos valores específicos obtidos (validação feita comparando-se os resultados obtidos com os comportamentos observados na prática).

\subsection{Diâmetro do eletrodo para emissão termiônica}

A Tabela 3 e a Tabela 4 mostram as densidades de corrente necessárias para emissão termiônica (Equação 1) às temperaturas de fusão/sublimação e ebulição, respectivamente, para cada material. Essas tabelas também mostram os diâmetros de eletrodo necessários para emissão a 100 e 300 A nas temperaturas citadas e para ângulos de ponta de 30, 60, 90 e $180^{\circ}$ (Equação 4). Em ambas as tabelas, a densidade de corrente resultante da emissão pelo Fe é a menor. Isso ocorre porque as temperaturas de fusão e ebulição do Fe são inferiores às dos outros materiais. Pode-se 
observar também que em todos os casos o diâmetro do eletrodo se reduz com o aumento da afiação, pois a área de emissão aumenta. Comparando-se a Tabela 3 com a Tabela 4, nota-se que o diâmetro de emissão de um mesmo material também pode ser reduzido com o aumento da temperatura de emissão.

Nas temperaturas de fusão do tungstênio e de sublimação da grafita, as densidades de corrente alcançadas por emissão termiônica são altas e os diâmetros suficientemente pequenos para qualquer corrente na faixa estudada. As pequenas dimensões viabilizam a utilização desses materiais como eletrodos com emissão termiônica em soldagem a arco (acessibilidade e portabilidade). Já para o ferro puro, a densidade de corrente é tão baixa que seria necessário um eletrodo da ordem de, no mínimo, $2 \mathrm{~m}$ de diâmetro (afiação de $30^{\circ}$ ) para haver a produção de $100 \mathrm{~A}$ na temperatura de fusão. Mesmo que a corrente desejada fosse reduzida para $10 \mathrm{~A}$, ainda seria necessário um eletrodo de aproximadamente $0,6 \mathrm{~m}$ para emitir a essa corrente à temperatura de fusão.

Com o aumento da temperatura de fusão para ebulição, o diâmetro necessário para emissão a $100 \mathrm{~A}$ se torna pontual para o W. Contudo, ainda seria necessário um eletrodo de $\mathrm{Fe}$ com diâmetro de no mínimo $3,24 \mathrm{~mm}$ com ponta totalmente exposta à temperatura de ebulição para emissão a $100 \mathrm{~A}$. Como não é possível manter a integridade física de um eletrodo de Fe nessas condições (além de uma grande área sujeita à evaporação na superfície cônica, pode-se inferir que, por causa do gradiente térmico, um grande volume da ponta do eletrodo estaria fundido) ele não poderia ser considerado não consumível.

A Figura 2 mostra os diâmetros de eletrodo com afiação de $30^{\circ}$ necessários para emissão a $100 \mathrm{~A}$ em função da temperatura, para diâmetros menores do que $16 \mathrm{~mm}$. Nota-se que o Fe, por ter menor função-trabalho, emite com mesma intensidade e mesma área de emissão (diâmetro) em temperaturas pouco menores em

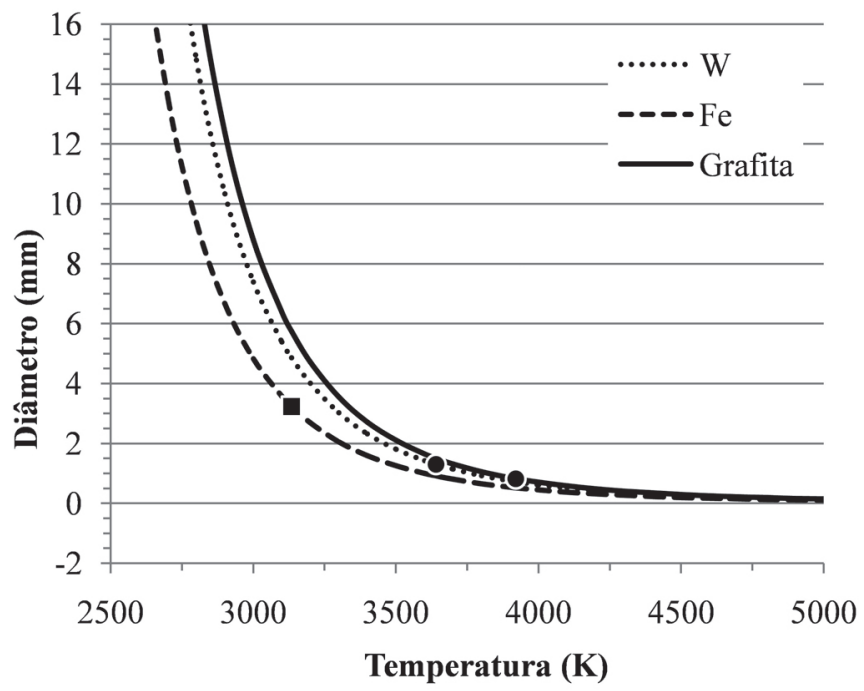

Figura 2. Diâmetro do eletrodo com afiação de $30^{\circ}$ necessário para emissão termiônica a $100 \mathrm{~A}$ em função da temperatura: • Temperaturas de fusão/sublimação do $\mathrm{C}$ e W e a temperatura de ebulição do Fe (as temperaturas de fusão do Fe e de ebulição do $\mathrm{W}$ estão fora da faixa de temperatura mostrada) relação à grafia e ao tungstênio. Contudo, apenas os eletrodos de grafita e W têm potencial para emitir à corrente desejada abaixo da temperatura de fusão com diâmetros suficientemente pequenos e apropriados à soldagem a arco. Por outro lado, um eletrodo de Fe precisaria estar a temperaturas muito superiores a sua temperatura de fusão (próximas a de ebulição) para ter dimensões adequadas à soldagem.

\subsection{Aquecimento máximo do eletrodo por efeito Joule}

No item anterior, foi calculado por simulação o diâmetro mínimo de eletrodo que levaria à emissão termiônica a 100 e 300 A em duas temperaturas. Mas, como explicado na metodologia, o eletrodo (material e diâmetro) precisaria ser aquecido até a temperatura de emissão pela passagem da corrente de trabalho. A Figura 3 apresenta a tendência de decrescimento do diâmetro do eletrodo necessário para emissão (eixo Y) em função da temperatura (eixo X), para a grafita, tungstênio puro e ferro puro (semelhante à Figura 2, mas contemplando toda a faixa de diâmetros e em escala logarítmica) sobrepostas à tendência de aumento da temperatura máxima alcançada pelos eletrodos após $1 \mathrm{~s}$ por efeito Joule (eixo X) em função da redução dos diâmetros (eixo Y).

Como mostrado na Figura 3, o aquecimento por efeito Joule se torna sensível para diâmetros menores do que $10 \mathrm{~mm}$ para os eletrodos de grafita e para diâmetros menores do que $1 \mathrm{~mm}$ para os eletrodos de tungstênio puro e ferro puro. As temperaturas máximas dos eletrodos de grafita com diâmetros muito inferiores a $10 \mathrm{~mm}$ são maiores do que $6000 \mathrm{~K}$ e não são contempladas na Figura 3. Um eletrodo de grafita de maiores dimensões (em relação às dimensões dos eletrodos dos outros materiais) consegue ser aquecido até a sua temperatura de emissão apenas por efeito Joule graças a sua alta resistividade elétrica, que supera a queda da resistência elétrica com o aumento da área de seção transversal (Equação 7). Já nos demais materiais estudados, o aquecimento resistivo até a temperatura de emissão só ocorre para diâmetros reduzidos, que favorece o aumento da resistência elétrica pela redução da área da seção transversal (Equação 7).

Ainda pela Figura 3, o cruzamento das curvas de aquecimento por efeito Joule (em função do diâmetro do eletrodo) com o diâmetro necessário para emissão (em função da temperatura) indica o diâmetro no qual é possível aquecer o eletrodo por efeito Joule até a temperatura de emissão sem que o arco apague. Eletrodos de diâmetros inferiores ao do cruzamento das curvas são facilmente elevados a temperaturas de emissão maiores que a necessária, podendo, contudo, o eletrodo sofrer maior desgaste. Por outro lado, eletrodos de diâmetros superiores não conseguirão ser totalmente aquecidos até temperaturas de emissão a tempo de evitar a extinção do arco. Neste caso, a emissão ainda é possível (como será discutido no terceiro parágrafo do item 3.5), mas não nas condições previstas na Figura 3 em que um eletrodo de maior área de emissão (maior diâmetro) consegue emitir em temperaturas menores.

Denomina-se aqui de diâmetro crítico $\left(\mathrm{d}^{*}\right)$ o maior diâmetro que pode ser aquecido por efeito Joule até a menor temperatura necessária para produzir a corrente desejada. Para que a temperatura de emissão no diâmetro crítico seja menor e, 
consequentemente, haja menor degradação do eletrodo, podemse utilizar materiais com alta resistividade elétrica, que aqueçam uma maior área até a temperatura de emissão (que é menor que a temperatura de emissão requerida por uma área menor), ou utilizar materiais com menor função-trabalho, que emitem com altas densidades de corrente mesmo a temperaturas mais baixas.

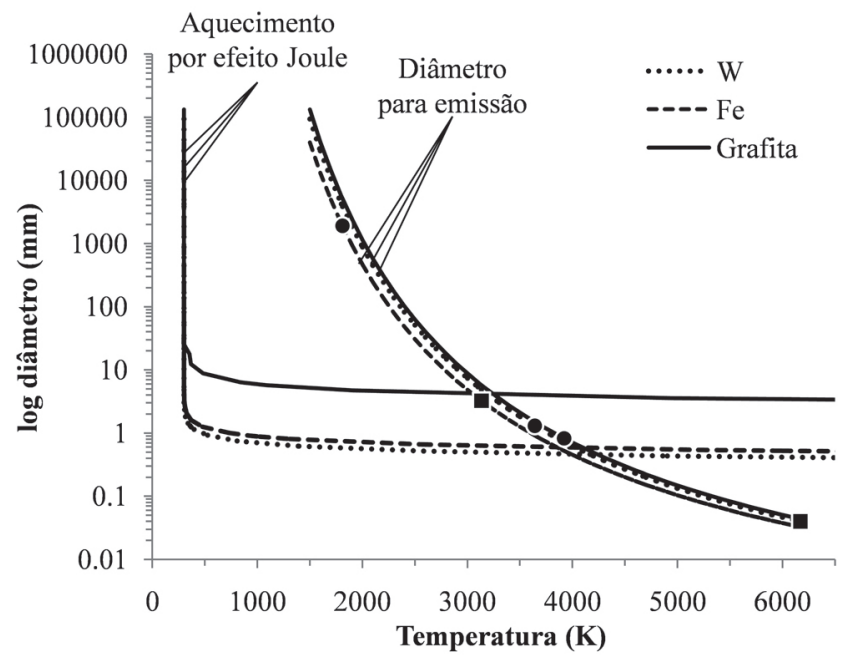

Figura 3. Temperatura alcançada pelo eletrodo devido ao aquecimento por efeito Joule em função do diâmetro e diâmetro necessário para emissão termiônica a $100 \mathrm{~A}$ em

função da temperatura, para eletrodos de grafita, W e Fe com afiação de $30^{\circ}$ (• Temperaturas de fusão/sublimação e ebulição dos materiais)

A Figura 4 apresenta uma ampliação da região da Figura 3 onde há o encontro das curvas de aquecimento por efeito Joule com as de diâmetro necessário para emissão termiônica a 100 A. A degradação do eletrodo será mínima se a temperatura de emissão no diâmetro crítico for inferior à temperatura de fusão do material. Apesar de o ferro puro ter potencial para emitir mais facilmente do que o tungstênio puro e a grafita, devido à sua menor função-trabalho, seu baixo ponto de fusão faz com que a densidade de corrente na fase sólida seja baixa e o diâmetro para emissão em nível de intensidade desejada seja muito alto. Além disso, um eletrodo de ferro puro não pode, em soldagem a arco, emitir elétrons correspondentes à corrente desejada na temperatura de fusão, pois não seria possível aquecer o eletrodo rapidamente por efeito Joule (lembrar que foi arbitrariamente assumido um tempo de $1 \mathrm{~s}$ para o aquecimento) até a temperatura de fusão devido às suas grandes dimensões e baixa resistividade. Contudo, áreas menores do eletrodo de ferro puro podem ser rapidamente aquecidas por efeito Joule a temperaturas elevadas, por causa do aumento da resistência elétrica devido à redução da área de seção transversal. Ainda assim, a temperatura de emissão no diâmetro crítico do $\mathrm{Fe}$ (que corresponde à máxima área aquecida por efeito Joule até a mínima temperatura necessária para emissão) seria superior à temperatura de ebulição. Apesar de uma pequena área exposta a temperaturas muito superiores à de ebulição em um eletrodo de Fe ser o suficiente para produção da corrente desejada, o gradiente de temperatura intrínseco ao eletrodo garante que uma área muito maior estará exposta a temperaturas iguais ou maiores do que à de ebulição em que a evaporação seria instantânea, impedindo, dessa forma que um eletrodo de Fe seja considerado como não consumível.

No caso do eletrodo de tungstênio puro, a emissão só ocorre a temperaturas superiores à temperatura de fusão, pois não é possível aquecer rapidamente (por efeito Joule) diâmetros maiores a temperaturas menores do que à de fusão. É razoável considerar que um eletrodo com apenas a sua superfície fundida seja não consumível se esta massa fundida se mantiver acoplada ao eletrodo. Neste caso, estariam atuando forças opostas à transferência de metal fundido, como por exemplo, a força devido à tensão superficial (principalmente) e as forças de vaporização. A própria presença da superfície fundida colabora na manutenção da integridade do eletrodo, já que resguarda a superfície não fundida do contato direto com a região catódica, onde há intensa geração de calor. Assim, apesar da perda da afiação com a fusão de um determinado volume na ponta do eletrodo, como pode acontecer na prática da soldagem TIG com eletrodo de $\mathrm{W}$ puro, é razoável admitir um eletrodo não consumível desse material exposto a temperaturas maiores do que a de fusão.

Eletrodos de grafita de diâmetros relativamente grandes conseguem ser aquecidos por efeito Joule até temperaturas de emissão inferiores à sua temperatura de sublimação, devido à alta resistividade da grafita. Esse material consegue emitir em temperaturas menores com áreas maiores. Apesar dos resultados indicarem que a emissão é possível a temperaturas inferiores à de sublimação da grafita, não se deve inferir que um eletrodo de grafita tenha maior durabilidade do que um de tungstênio puro. Enquanto eletrodos de W normalmente operam em atmosfera parcial ou totalmente inerte, eletrodos de grafita operam em atmosfera oxidante sem qualquer tipo de proteção gasosa. $\mathrm{O}$

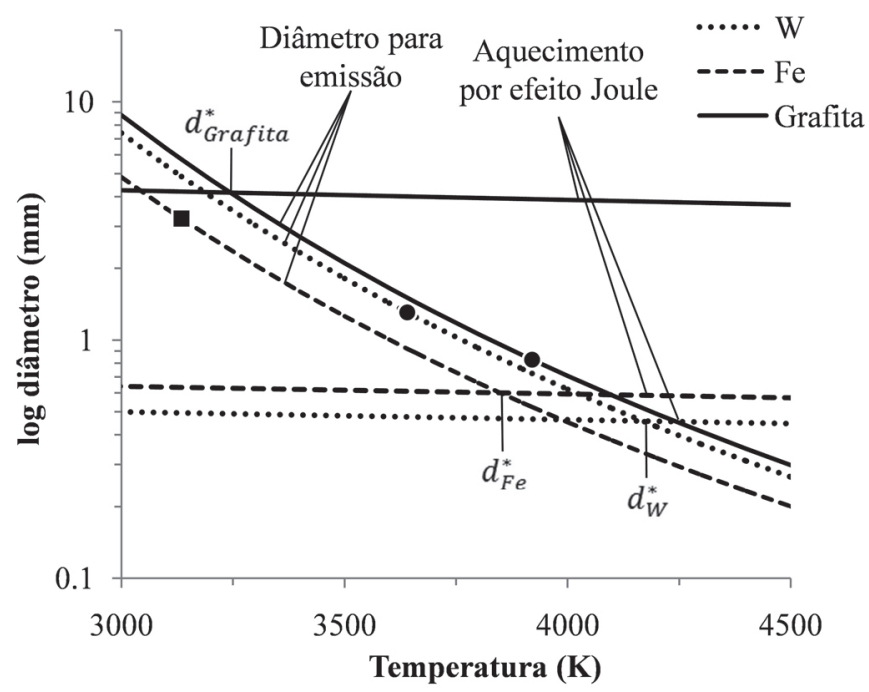

Figura 4. Diâmetro crítico dos materiais $\left(\mathrm{d}^{*}\right)$ indicando o maior diâmetro de eletrodo com afiação de $30^{\circ}$ que pode ser aquecido por efeito Joule até a temperatura de emissão: • Temperatura de fusão/sublimação do $\mathrm{W}$ e C e $\boldsymbol{\Xi}$ temperatura de ebulição do $\mathrm{Fe}$ (as temperaturas de fusão do Fe e de ebulição do W estão fora da faixa de temperatura mostrada) 
Tabela 5. Densidade de corrente e diâmetro de eletrodo necessário para emissão termiônica a 100 e 300 A na temperatura de fusão do $\mathrm{W}$ e do $\mathrm{W}+2 \% \mathrm{ThO}_{2}$

\begin{tabular}{|c|c|c|c|c|c|c|c|c|c|}
\hline & & \multicolumn{4}{|c|}{$\mathbf{1 0 0} \mathbf{A}$} & \multicolumn{4}{|c|}{$300 \mathbf{A}$} \\
\hline & $\mathbf{J}$ & $\mathbf{d}_{\mathbf{3 0}^{\circ}}$ & $\mathbf{d}_{\mathbf{6 0}^{\circ}}$ & $\mathbf{d}_{\mathbf{9 0}^{\circ}}$ & $\mathbf{d}_{\mathbf{1 8 0}^{\circ}}$ & $\mathbf{d}_{\mathbf{3 0}^{\circ}}$ & $\mathbf{d}_{\mathbf{6 0}^{\circ}}$ & $\mathbf{d}_{\mathbf{9 0}^{\circ}}$ & $\mathbf{d}_{\mathbf{1 8 0 ^ { \circ }}}$ \\
\hline $\mathbf{W}$ & $1,93 \mathrm{E}+07$ & 1,31 & 1,82 & 2,16 & 2,57 & 2,26 & 3,15 & 3,74 & 4,45 \\
\hline $\mathbf{T h O}_{2}$ & $6,95 \mathrm{E}+09$ & 0,07 & 0,10 & 0,11 & 0,14 & 0,12 & 0,17 & 0,20 & 0,23 \\
\hline
\end{tabular}

$J$ - Densidade de corrente (A. $\left.\mathrm{m}^{-2}\right) ; d$ - diâmetro de eletrodo (mm)

contato direto do carbono (do eletrodo de grafita) com o oxigênio (do ambiente ou residual de fabricação do eletrodo) associado às altas temperaturas levam à formação de gases como $\mathrm{CO}$ e $\mathrm{CO}_{2}$, acelerando o consumo do eletrodo. Além disso, no eletrodo de grafite não há formação de uma superfície fundida entre a região catódica, onde as temperaturas são mais elevadas, e o eletrodo sólido que atue no sentido de retardar a fusão do eletrodo, como ocorre com o eletrodo de W.

Enquanto eletrodos de $\mathrm{W}$ e grafita, aquecidos por efeito Joule, emitiriam acima da temperatura de fusão e abaixo da temperatura de sublimação, respectivamente, um eletrodo de $\mathrm{Fe}$ precisaria ter temperatura superficial maior que a de ebulição, condição em que não se pode manter sua integridade física. Mesmo que se considerasse a temperatura de ebulição como a temperatura limite para emissão (Tabela 4) o diâmetro de eletrodo necessário para emissão nesta temperatura não seria suficientemente aquecido por efeito Joule a tempo de evitar a extinção do arco.

\subsection{Efeito da dopagem com óxidos facilitadores de emissão}

A dopagem do tungstênio com tória reduz a funçãotrabalho (Tabela 1) e a emissão termiônica nesses eletrodos é facilitada, ou seja, pode-se emitir em uma mesma intensidade de corrente com temperaturas mais baixas ou com diâmetros menores. A Tabela 5 apresenta o diâmetro mínimo de eletrodo de $\mathrm{W}+2 \% \mathrm{ThO}_{2}$ necessário para emissão termiônica a 100 e 300 A na temperatura de fusão, em comparação com o eletrodo de W puro. Como a densidade de corrente produzida é muito maior no eletrodo dopado, o diâmetro de eletrodo mínimo necessário é ainda mais reduzido em relação ao do eletrodo de tungstênio puro. A Figura 5 mostra a comparação entre o aquecimento por efeito Joule e o diâmetro necessário para emissão pelos eletrodos de $\mathrm{W}$ puro e de $\mathrm{W}+2 \% \mathrm{ThO}_{2}$. $\mathrm{O}$ aquecimento por efeito Joule dos dois eletrodos é praticamente o mesmo, mas a emissão no eletrodo dopado é possível em temperaturas menores que a de fusão, enquanto que no tungstênio puro a emissão ocorre apenas em temperaturas superiores à de fusão. Como o eletrodo dopado com tória emite abaixo da temperatura de fusão, é possível que haja uma pequena faixa de diâmetros menores do que o crítico cuja utilização implique em aumento da temperatura de emissão, mas sem ainda ultrapassar a temperatura de fusão e aumentar o desgaste do eletrodo. Esta proposição justifica mais uma evidência prática de que, ao contrário de eletrodos de $\mathrm{W}$ puro, eletrodos dopados não apresentam fusão da ponta afiada no processo TIG.

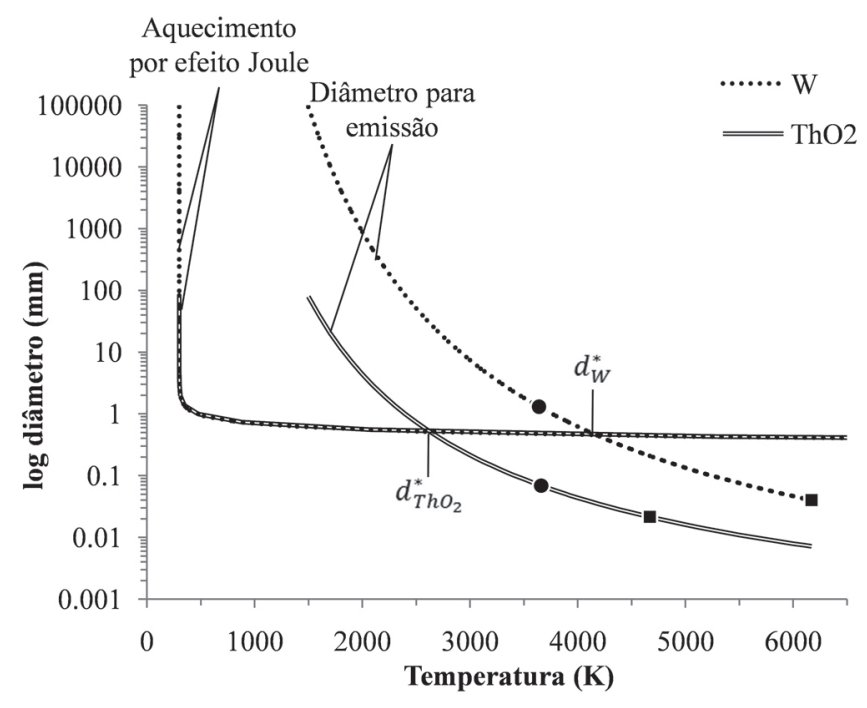

Figura 5. Temperatura alcançada por um eletrodo devido ao aquecimento por efeito Joule em função do diâmetro e diâmetro necessário para emissão termiônica a $100 \mathrm{~A}$ em função da temperatura, para eletrodos de $\mathrm{W}$ puro e $\mathrm{W}+2 \% \mathrm{ThO}_{2}$ com afiação de $30^{\circ}$ (• Temperaturas de fusão/sublimação e $\boldsymbol{\bullet}$ ebulição dos materiais)

\subsection{Validação dos resultados frente ao comportamento observado na Prática}

Para todos os materiais foram calculados os diâmetros mínimos necessários para obtenção da corrente desejada em determinadas temperaturas por emissão termiônica. Observou-se que para produção de maiores correntes é necessário maior área de emissão e, consequentemente, maior diâmetro de eletrodo, o que é coerente com os saberes referentes à soldagem TIG a fim de evitar o desgaste precoce do eletrodo.

Como resultado desse trabalho também foi observado que, para emitir na intensidade de corrente desejada, a redução do diâmetro do eletrodo implica em aumento da temperatura necessária para emissão. Na prática, a utilização de um eletrodo de diâmetro menor do que o recomendado para uma determinada corrente leva a um maior aquecimento do eletrodo por efeito Joule resultando em maior densidade de corrente produzida e desgaste mais acelerado.

Contudo, os resultados desse trabalho indicam que o aumento do desgaste do eletrodo com a redução do diâmetro depende da temperatura mínima com que o material consegue emitir e 
de sua proximidade da temperatura de fusão (que é o caso do eletrodo de $\mathrm{W}+2 \% \mathrm{ThO}_{2}$, ilustrado pela Figura 5).

Observou-se ainda que em processos de soldagem a arco não é possível diminuir a temperatura de emissão com o aumento da área de emissão (aumento do diâmetro do eletrodo), pois não é possível aquecer dimensões maiores do que as do diâmetro crítico em tempo suficientemente curto para garantir a abertura do arco. Tal comportamento se verifica na prática na utilização de um eletrodo de diâmetro maior do que o recomendado, em que a região de contato arco-eletrodo não se expande com o aumento da área disponível para emissão, mas permanece restrito à maior área (na extremidade da ponta do eletrodo) que consegue ser e permanecer aquecida.

Ressalta-se que o eletrodo de tungstênio puro emite em temperaturas superiores a de fusão, que leva a perda de afiação, e que eletrodos de tungstênio dopados com tória emitem abaixo da temperatura de fusão e mantém, por esse motivo, sua afiação e maior durabilidade. Esses resultados estão também coerentes com resultados experimentais reportados na literatura, por exemplo, por Tanaka [14] e Ushio [15]. Na prática, a redução de calor gerado nos acoplamentos, que faz com que o eletrodo dopado opere em temperatura inferior a do eletrodo de $\mathrm{W}$ puro, se reflete na queda de tensão de trabalho.

Em soldagem TIG verifica-se a necessidade da afiação da ponta do eletrodo para abertura e manutenção de um arco estável. Tal prática pode ser justificada por duas linhas de raciocínio abordadas neste trabalho. No eletrodo afiado, o início da emissão é facilitado tanto pelo maior aquecimento resistivo da ponta, por ter menor área de seção transversal quanto pela menor perda de calor por condutividade térmica. Já a manutenção de um arco estável está associada à sua capacidade de se adaptar às diversas intensidades de corrente requeridas sem sofrer maiores perturbações do fluxo do gás. Variações na intensidade de corrente requerem variações na área de emissão (Equação 4). No eletrodo afiado, a área de emissão varia continuamente com o diâmetro, permitindo que o arco se adapte sem sofrer variações bruscas e interferência do fluxo de gás.

Finalmente, demonstrou-se a inviabilidade da utilização do Fe como eletrodo não consumível em processos de soldagem a arco. Inviabilidade esta também inferida da prática, pela ausência de eletrodos não consumíveis de ferro e suas ligas, nos quais a emissão é prioritariamente por campo.

Desta forma, apesar das simplificações adotadas para os cálculos, os resultados obtidos neste trabalho quanto às temperaturas de emissão e durabilidade dos eletrodos estão coerentes com a prática do processo TIG e resultados reportados na literatura. Fica então como sugestão, como outro desafio tecnológico, que pesquisadores aperfeiçoem o modelo apresentado neste trabalho pela minimização das simplificações adotadas, visando aumentar a acurácia dos resultados e até possibilitar novos estudos sobre a utilização de diferentes óxidos ou elementos na dopagem dos eletrodos de W.

\section{Conclusões}

Apesar do $\mathrm{Fe}$ conseguir emitir termionicamente com intensidades de corrente adequadas à soldagem na temperatura de fusão, as grandes dimensões requeridas de um eletrodo desse material (da ordem de metros) com essa finalidade comprometeriam a acessibilidade e portabilidade do conjunto tocha-peça. Além disso, um eletrodo de Fe nessas condições não poderia ser totalmente aquecido à temperatura de fusão apenas por efeito Joule a tempo de iniciar um arco por emissão termiônica. Por outro lado, um eletrodo de Fe de menor diâmetro e tamanho apropriado à soldagem conseguiria ser aquecido por efeito Joule às temperaturas de emissão, mas essas temperaturas precisariam ser superiores a sua temperatura de ebulição para obtenção da corrente desejada. Dessa forma, apesar das simplificações adotadas nos cálculos, ficam justificadas as razões pelas quais materiais como o $\mathrm{Fe}$, que, apesar de conseguirem emitir termionicamente, não podem ser usados como materiais para eletrodos não consumíveis de soldagem a arco.

Por outro lado, a aplicação da mesma abordagem analítica ao $\mathrm{W}$ puro e ao $\mathrm{W}+2 \% \mathrm{ThO}_{2}$ mostrou que diâmetros na ordem de grandeza de décimos de milímetros seriam suficientes para garantir a emissão correspondente à corrente desejada por efeito termiônico a temperaturas superiores à de fusão (mas menores do que a de ebulição), para o $\mathrm{W}$ puro, e inferiores à de fusão, para o $\mathrm{W}+2 \% \mathrm{ThO}_{2}$. Esta é a razão para o eletrodo de $\mathrm{W}$ puro perder mais facilmente a afiação do que o eletrodo dopado.

Além disto, o procedimento usual de afiação da ponta do eletrodo para abertura e manutenção de um arco estável na soldagem TIG pode ser justificado pela maior facilidade no inicio da emissão com o maior aquecimento resistivo e menor perda de calor por condução na ponta do eletrodo, pela adaptação sutil do arco às variações de área requeridas por diferentes solicitações de intensidade de corrente e pelo fato da geometria da ponta minimizar as perturbações impostas pelo fluxo de gás sobre o arco.

O potencial teórico da geração de corrente adequada à soldagem por emissão termiônica também foi atestado para a grafita. Observou-se que eletrodos de poucos milímetros de diâmetro seriam suficientes para garantir a emissão em níveis usuais de correntes abaixo da temperatura de sublimação da grafita.

Dessa forma, diferentemente do Fe, o W puro, dopado e a grafita podem ser considerados e utilizados como eletrodos não consumíveis em processos de soldagem a arco.

\section{Agradecimentos}

Os autores do trabalho gostariam de agradecer à CAPES, pela bolsa de pós-graduação, e ao CNPQ, pela bolsa de pesquisa, assim como aos programas de pós-graduação da Universidade Federal do Paraná, PIPE e PG-Mec, pela oportunidade.

\section{Referências Bibliográficas}

[1] CHENG, D. Y., Dynamics of arc ignition and cathode spot movement of thermionically emitting cathode surfaces. Journal of Applied Physics. USA, v. 41, n. 9, p. 3626, August, 1970.

[2] LIMA, E. F.; FOSCHINI, M.; MAGINI, M., O efeito termoiônico: uma nova proposta experimental. Revista Brasileira de Ensino de Física, Brasil, v. 23, n. 4, p.391-394, 
Dezembro, 2001.

[3] ANDRADE, N. S. et al., Investigação teórica e experimental do efeito termiônico. Revista Brasileira de Ensino de Física, Brasil, v. 35, n. 1, p. 1308, Fevereiro, 2013.

[4] RICHARDSON, O. W., Thermionic phenomena and the laws which govern them. Nobel Lecture, December, 1929. Disponível em: <http://openx.nobelprize.org/nobel_prizes/ physics/laureates/1928/richardson-lecture.pdf $>$. Acesso em: Junho, 2013.

[5] GUILE, A. E., Arc-electrode phenomena. Proc. IEE, IEE Reviews, v. 118, n. 9, p. 1131-1154, September, 1971.

[6] LANCASTER, J. F., The Physics of Welding. $2^{\text {nd }}$ ed, Oxford: Pergamon press, 1986, p. 124-125.

[7] DUSHMAN, S., Electron Emission from metals electron emission from metals as a function of temperature. Phys. Rev. v. 21, p. 623-636, June, 1923.

[8] Propriedades do Tungstênio. Disponível em: $<$ http://www. matweb.com/search/DataSheet.aspx?MatGUID $=41 \mathrm{e} 0851 \mathrm{~d} 2 \mathrm{f} 3 \mathrm{c}$ 417ba69ea0188fa570e3>. Acesso em: Junho, 2013.

[9] JAIN, S. C.; KRISHNAN, K. S., The thermionic constants of metals and semi-conductors. I. Graphite. Proceedings of the Royal Society of London. Series A, Mathematical and Physical Sciences, v. 213, n. 1113, p. 143-157, June, 1952.

[10] Propriedades da Grafita. Disponível em: $<$ http://www. matweb.com/search/datasheet.aspx?MatGUID=3f64b98540244 5c0a5af911135909344>. Acesso em: Junho, 2013.

[11] JAIN, S. C.; KRISHNAN, K. S., The thermionic constants of metals and semi-conductors. II. Metals of the first transition group. Proc R Soc A, v. 215, p. 431-437, 1952.

[12] Propriedades do Ferro. Disponível em: <http://www. matweb.com/search/DataSheet.aspx?MatGUID $=654 \mathrm{ca} 9 \mathrm{c} 35826$ 4b5392d43315d8535b7d >. Acesso em: Junho, 2013.

[13]KENTARO, Y.; SHINICHI, T.; MANABU, T., Numerical Analysis of Current Attachment at Thermionic Cathode for Gas Tungsten Arc at Atmospheric Pressure. Transactions of JWRI, v. 38, n. 1, 2009.

[14] Propriedades do Óxido de Tório. Disponível em: $<$ http:// www.matweb.com/search/DataSheet.aspx?MatGUID $=\mathrm{db} 32 \mathrm{~b} 3$ 96093d446aa4206468f0681736\&ckck=1>. Acesso em: Junho, 2013.

[15] F. RIGHINI et al., Thermophysical Properties of Thoriated Tungsten Above $3600 \mathrm{~K}$ by a Pulse-Heating Method. International Journal of Thermophysics, vol. 17, n. 5, 1996.

[16] M. TANAKA et al., In Situ Measurements of Electrode Work Functions in Free-Burning Arcs During Operation at Atmospheric Pressure. Journal of Physics D: Applied Physics, vol. 29, p. 29-35, 2005.

[17] M. USHIO, Arc discharge and electrode phenomena, Pure \& Applied Chemistry, vol. 60, n. 5, p. 809-814, 1988. 